\title{
Divergences of Discrete States Amplitudes and Effective Lagrangian in 2D String Theory.
}

\author{
I.Ya.Aref'eva * \\ and \\ A.P.Zubarev ${ }^{\dagger}$
}

May, 1992

\begin{abstract}
Scattering amplitudes for discrete states in 2D string theory are considered. Pole divergences of tree-level amplitudes are extracted and residues are interpreted as renormalized amplitudes for discrete states. An effective Lagrangian generating renormalized amplitudes for open string is written and corresponding Ward identities are presented. A relation of this Lagrangian with homotopy Lie algebra is discussed.
\end{abstract}

SMI $-2-92$

*Steklov Mathematical Institute, Vavilov 42, GSP-1, 117966, Moscow, Russia

${ }^{\dagger}$ Supported in part by Moscow Physical Society Grant 


\section{Introduction}

Recently Witten and Zwiebach [1] and E.Verlinde [2] have considered Ward identities for discrete states amplitudes [3]- [11] in 2D string theory. These identities express a symmetry which is important in understanding of background independent formulation of the corresponding string field theory. It was noted [1] a relation of these Ward identities with homotopy Lie algebra [16].

In this note we consider these amplitudes in more details. In fact correlation functions for discrete states diverge because external momentum coincide with poles of amplitudes. We use an analytic regularization and interpret a residue with respect to regularization parameter $\epsilon$ as renormalized correlation functions for discrete states. These correlation functions satisfy Ward identities. Such renormalization corresponds to renormalization of coupling constant and wave function in string field theory [8] and it should correspond to renormalization of the Verlinde master equation [2].

We present an effective Lagrangian generating renormalized correlation function. It turns out that this Lagrangian is related with the structure of the homotopy Lie algebra.

\section{Tree level N-point amplitudes}

Below we carry out the calculations of the simplest on-shell amplitudes for discrete states. From this results it is not hard to infer the general structure.

Let us consider the tree level 4-point open string amplitude for discrete states. Assume that three of them are $(+)$ states

$$
Y_{s_{i}, n_{i}}^{+}=c W_{s_{i}, n_{i}}^{+}
$$

$i=1,2,3$, and the fourth is $(-)$ state

$$
Y_{s_{i}, n_{i}}^{-}=c W_{s_{i}, n_{i}}^{-}
$$

$i=4$, where

$$
\begin{gathered}
W_{s, s-n}^{ \pm}=\sqrt{(2 s-n) ! / n !(2 s) !} \underbrace{\left[H^{-}, \ldots\left[H^{-}\right.\right.}_{n}, W_{s, s}^{ \pm}] \ldots], \\
H^{-}=\oint \frac{d z}{2 \pi i} e^{-i \sqrt{2} X}(z), \quad W_{s, s}^{ \pm}=e^{i \sqrt{2} s X} e^{\sqrt{2}(1 \mp s) \phi},
\end{gathered}
$$

$s$ is positive integer or half integer.

In this case we have

$$
\begin{gathered}
\mathcal{A}_{4}\left(\left(s_{1}, n_{1}\right)^{+}\left(s_{2}, n_{2}\right)^{+}\left(s_{3}, n_{3}\right)^{+}\left(s_{4}, n_{4}\right)^{-}\right)= \\
\int<Y_{s_{1}, n_{1}}^{+}\left(z_{1}\right) Y_{s_{2}, n_{2}}^{+}\left(z_{2}\right) Y_{s_{3}, n_{3}}^{+}\left(z_{3}\right) \oint b(z) d z Y_{s_{4}, n_{4}}^{-}\left(z_{4}\right)>d \mu\left(z_{3}\right) .
\end{gathered}
$$

These amplitudes contain divergences. For example, the amplitude for four tachyon states with exceptional momentum is ill-defined,

$$
\begin{gathered}
\mathcal{A}_{4}\left((1,1)^{+}(1,-1)^{+}(1,1)^{+}(1,-1)^{-}\right)= \\
=\int d z<\left(Y_{1,1}^{+}\right)(\infty)\left(Y_{1,-1}^{+}\right)(1)\left(W_{1,1}^{+}\right)(z)\left(Y_{1,-1}^{-}\right)(0)>=\int_{0}^{1} d z \frac{1}{z^{2}(1-z)^{2}} .
\end{gathered}
$$


The integral is divergent and one should use some regularization procedure. Results will depend on the regularization. We use a special analytical regularization and corresponding integral understand in sense of the analytical continuation

$$
r e g_{\epsilon}\left(\int_{0}^{1} d z z^{-n}(1-z)^{-m}\right)=\int_{0}^{1} d z z^{-n-\epsilon}(1-z)^{-m-\epsilon}=B(1-n-\epsilon, 1-m-\epsilon),
$$

Note that just with such analytical continuation on external momentum one deals in the usual $\mathrm{D}=26$ critical bosonic string. Under this convention the amplitude (5) has a pole with respect to the regularization parameter

$$
\mathcal{A}_{4}\left((1,1)^{+}(1,-1)^{+}(1,1)^{+}(1,-1)^{-}\right) \sim \frac{2}{\epsilon}
$$

Some amplitudes (幽) under regularization (6) have not singularities. For example, the amplitude for four vector states is finite

$$
\begin{gathered}
\mathcal{A}_{4}\left((1,0)^{+}(1,0)^{+}(1,0)^{+}(1,0)^{-}\right)= \\
=\int_{0}^{1}<(c \partial x)(\infty)(c \partial x)(1)(\partial x)(z)(c \partial x)(0) e^{2 \sqrt{2} \phi}>=\int_{0}^{1} d z\left[\frac{1}{z^{2}}+\frac{1}{(1-z)^{2}}+1\right]=-1 .
\end{gathered}
$$

It is easy to see that the leading singularities on $\epsilon$ can be extracted by inserting the sum over cohomology classes with ghost number 1 (physical states) in the correlation functions (14). Indeed, to calculate the s-channel amplitude (4) let us perform the operator product expansion of operators $W_{s_{3}, n_{3}}^{+}(z)$ and $Y_{s_{4}, n_{4}}^{-}(0)$,

$$
W_{s_{3}, n_{3}}^{+}(z) Y_{s_{4}, n_{4}}^{-}(0)=\sum_{i} \frac{1}{z^{i}} \mathcal{O}_{i}(0) .
$$

Physical states $Y_{s^{\prime}, n^{\prime}}^{-}$(non-trivial cohomology classes) appeared in $\mathcal{O}_{1}$ due to the OPE of operators $W^{+}$and $W^{-}$, see [12], give leading singularities (l.s.) and we can write

$$
\mathcal{A}_{4}^{l . s .}=\sum_{s^{\prime}, n^{\prime}} \int_{0}^{1} \frac{d z}{z^{1+\epsilon}}<Y_{s_{1}, n_{1}}^{+}(\infty) Y_{s_{2}, n_{2}}^{+}(1) Y_{s^{\prime},-n^{\prime}}^{-}(0)><Y_{s^{\prime}, n^{\prime}}^{+}(\infty) Y_{s_{3}, n_{3}}^{+}(z) Y_{s_{4}, n_{4}}^{-}(0)>
$$

Therefore we have shown that the 4-point amplitude under the special analytical regularization has the pole form

$$
\mathcal{A}_{4}=\frac{\tilde{\mathcal{A}}_{4}}{\epsilon}+O(1)
$$

Just this function $\tilde{\mathcal{A}}_{4}$ we want interpret as the 4-point correlation function for discrete states. The physical meaning of the above calculations is clear, since we do nothing but extract the residue of $s$-channel pole.

Moreover, $\tilde{\mathcal{A}}_{4}=\operatorname{Res} \mathcal{A}_{4}$ can be expressed in terms of 3-point functions (10). 3-point functions have been considered in [12] and have the form

$$
\begin{gathered}
<Y_{s_{1}, n_{1}}^{+} Y_{s_{2}, n_{2}}^{+} Y_{s_{3}, n_{3}}^{-}>=f_{s_{1} n_{1}, s_{2} n_{2}}^{s_{3} n_{3}} \\
f_{s_{1} n_{1}, s_{2} n_{2}}^{s_{3} n_{3}}=\delta_{s_{1}+s_{2}-1, s_{3}} \delta_{n_{1}+n_{2},-n_{3}} \tilde{f}_{s_{1} n_{1}, s_{2} n_{2}}
\end{gathered}
$$


For integers $s_{1}$ and $s_{2}$ one has $\tilde{f}_{s_{1} n_{1}, s_{2} n_{2}}=s_{2} n_{1}-s_{1} n_{2}$. In (12) the following changing of the normalization of the discrete states $Y_{s, n}^{+} \rightarrow 1 / N(s, n) Y_{s, n}^{+}, \quad \tilde{N}(s, n)=$ $-\sqrt{s / 2}(2 s-1) ! N(s, n), \quad N(s, n)=\sqrt{(s+m) !(s-m) ! /(2 s-1) !}$ is assumed. The half integer indices $s$ bring some subtlety, but in all cases the symmetry properties of $\tilde{f}$ can be written as

$$
\tilde{f}_{s_{1} n_{1}, s_{2} n_{2}}=-(-1)^{2\left(m_{1} m_{2}-s_{1} s_{2}+s_{1}+s_{2}\right)} \tilde{f}_{s_{2} n_{2}, s_{1} n_{1}} .
$$

Now we are going to show that analogues structure takes place for $N$-point tree correlation functions for arbitrary $N>3$. In $s$-channel we have

$$
\begin{array}{r}
\mathcal{A}_{N}\left(\left(s_{1}, n_{1}\right)^{+}\left(s_{2}, n_{2}\right)^{+} \ldots\left(s_{N-1}, n_{N-1}\right)^{+}\left(s_{N}, n_{N}\right)^{-}\right)=\int_{0}^{1} d z_{1} \int_{0}^{z_{1}} d z_{2} \ldots \\
\int_{0}^{z_{N-4}} d z_{N-3}<Y_{s_{1}, n_{1}}^{+}(\infty) Y_{s_{2}, n_{2}}^{+}(1) W_{s_{3}, n_{3}}^{+}\left(z_{1}\right) \ldots W_{s_{N-1}, n_{N-1}}^{+}\left(z_{N-3}\right) Y_{s_{N}, n_{N}}^{-}(0)>
\end{array}
$$

Performing the OPE as in (9) we get

$$
\begin{gathered}
\mathcal{A}_{N}^{l . s .}\left(\left(s_{1}, n_{1}\right)^{+}\left(s_{2}, n_{2}\right)^{+} \ldots\left(s_{N-1}, n_{N-1}\right)^{+}\left(s_{N}, n_{N}\right)^{-}\right)= \\
\int_{0}^{1} \frac{d z_{1}}{z_{1}} \int_{0}^{z_{1}} \frac{d z_{2}}{z_{2}} \ldots \int_{0}^{z_{N-4}} \frac{d z_{N-3}}{z_{N-3}} \sum_{s^{(1)}, n^{(1)}} \ldots \sum_{s^{(N-3)} n^{(N-3)}}<Y_{s_{1}, n_{1}}^{+} Y_{s_{2}, n_{2}}^{+} Y_{s^{(1)},-n^{(1)}}^{-}> \\
<Y_{s^{(1)}, n^{(1)}}^{+} Y_{s_{3}, n_{3}}^{+} Y_{s^{(2)},-n^{(2)}}^{-}>\ldots<Y_{s^{(N-3)}, n^{(N-3)}}^{+} Y_{s_{N-1}, n_{N-1}}^{+} Y_{s_{N}, n_{N}}^{-}>.
\end{gathered}
$$

So under the analytical prescription (10) we get the following answer

$$
\mathcal{A}_{N}^{l . s .}\left(\left(s_{1}, n_{1}\right)^{+}\left(s_{2}, n_{2}\right)^{+} \ldots\left(s_{N-1}, n_{N-1}\right)^{+}\left(s_{N}, n_{N}\right)^{-}\right)=\frac{1}{\epsilon^{N-3}} \tilde{\mathcal{A}}
$$

Therefore the $N$-point amplitude for $(N-1)(+)$ states and one $(-)$ state after renormalization can be represented as

$$
\tilde{\mathcal{A}}(N-1,1)=\sum_{s^{(1)}, n^{(1)}} \ldots \sum_{s^{(N-3), n^{(N-3)}}} f_{s_{1} n_{1}, s_{2} n_{2}}^{s^{(1)}-n^{(1)}} f_{s^{(1)} n^{(1)}, s_{3} n_{3}}^{s^{(2)}} \ldots f_{s^{(N-3)} n^{(N-3)}, s_{N-1} n_{N-1}}^{s_{N} n_{N}} .
$$

One has a factorisation of the $N$-point amplitude in terms of 3-point one. The divergences of discrete states amplitudes can be removed by renormalization of wave function and coupling constant in the string field theory approach

$$
\Phi \rightarrow \mathcal{Z}_{1} \Phi, \quad \mathcal{Z}_{1}=\epsilon^{1 / 2}
$$

where $\Phi$ is a string field,

$$
g \rightarrow g \mathcal{Z}_{0}, \quad \mathcal{Z}_{0}=\epsilon^{-3 / 2}
$$

We perform the momentum-independent renormalization. It is interesting to note that under this renormalization the tachyon correlation functions with non-exceptional momentum (non-compactified correlation functions) vanish and only correlation functions for discrete momentum $T_{n \sqrt{2}}$ survey. Our renormalization for tachyon fields with exceptional momentum coincides with momentum-dependent renormalization [6] up to some normalization factors. 


\section{Renormalized Ward identities and effective Lagrangian}

From the currents $W$ one can form the charges [10

$$
Q_{s, m}^{ \pm}=\oint \frac{d z}{2 \pi i} W_{s, m}^{ \pm}(z)
$$

These charges form an algebra

$$
\left[Q_{s_{1}, m_{1}}^{+}, Q_{s_{2}, m_{2}}^{+}\right]_{ \pm}=\tilde{f}_{s_{1} m_{1}, s_{2} m_{2}} Q_{s_{1}+s_{2}-1, m_{1}+m_{2}}^{+}
$$

where \pm is in accordance with (13). This symmetry was observed in the context of matrix models [14].

The current conservation $\bar{\partial} W=0$ implies the symmetry Ward identities on correlation functions

$$
\int<Q_{s, m}^{ \pm} Y_{s_{1}, m_{1}}^{+} \ldots Y_{s_{N}, m_{N}}^{-} \prod \oint b>=0
$$

where three of vertex operators $Y^{ \pm}$are fixed and the rest integrated. The OPE's $W_{s, m}^{+}$ and $Y_{s_{1}, m_{1}}^{ \pm}$assumes the formal Ward identities

$$
\begin{gathered}
\tilde{f}_{s m, s_{1} m_{1}} \int<Y_{s_{1}+s-1, m_{1}+m}^{+} Y_{s_{2}, m_{2}}^{+} \ldots Y_{s_{N}, m_{N}}^{-} \prod \oint b> \pm \\
\tilde{f}_{s m, s_{2} m_{2}} \int<Y_{s_{1}, m_{1}}^{+} Y_{s_{2}+s-1, m_{2}+m}^{+} \ldots Y_{s_{N}, m_{N}}^{-} \prod \oint b> \pm \ldots \\
\pm \tilde{f}_{s-s_{N}+1-m-m_{N}, s_{N} m_{N}} \int<Y_{s_{1}, m_{1}}^{+} Y_{s_{2}, m_{2}}^{+} \ldots Y_{s_{N}-s+1, m_{N}+m}^{-} \prod \oint b>=0
\end{gathered}
$$

However all correlation functions in (24) are ill-defined. After renormalization (19) we left with the correlation functions (18) which obvious satisfy the tree-level Ward identities

$$
\begin{gathered}
\tilde{f}_{s m, s_{1} m_{1}} \tilde{\mathcal{A}}\left(\left(s_{1}+s, m_{1}+m\right)^{+}\left(s_{2}, m_{2}\right)^{+}, \ldots\left(s_{N}, m_{N}\right)^{-}\right) \pm \\
\tilde{f}_{s m, s_{2} m_{2}} \tilde{\mathcal{A}}\left(\left(s_{1}, m_{1}\right)^{+}\left(s_{2}+s, m_{2}+m\right)^{+}, \ldots\left(s_{N}, m_{N}\right)^{-}\right) \pm \ldots \pm \\
\tilde{f}_{s-s_{N}+1}-m-m_{N}, s_{N} m_{N} \tilde{\mathcal{A}}\left(\left(s_{1}, m_{1}\right)^{+}\left(s_{2}, m_{2}\right)^{+}, \ldots\left(s_{N}-s+1, m_{N}+m\right)^{-}\right)=0 .
\end{gathered}
$$

So far we have found that Ward identities are preserved under the renormalization.

There are additional subtleties in the closed string related with that one expect the symmetry charges to alter the particle number [1, 2, 15].

One can write an effective Lagrangian which generates the renormalized tree amplitudes (18). This Lagrangian describes an interaction of two independent fields $\Phi_{s, n}$ and $\bar{\Phi}_{s, n}$ with indices $s \geq 0,-s \leq n \leq s$. The Lagrangian has the form

$$
\mathcal{L}(\Phi, \bar{\Phi})=\sum_{a} \bar{\Phi}_{a} \Phi^{a}+g \sum_{a, b, c} \Phi^{a} \Phi^{b} \bar{\Phi}_{c} f_{a b}{ }^{c},
$$

where $\Phi^{a}=\Phi_{s, n}$ and $\bar{\Phi}_{a}=\bar{\Phi}_{s,-n}$ and

$$
f_{a b}^{c}=<Y_{a}^{+} Y_{b}^{+} Y_{c}^{-}>
$$

The fields $\Phi_{s, n}$ and $\bar{\Phi}_{s, n}$ for integer $s$ anticommute and for half integer $s$ their statistic is in accordance with symmetry properties (13). It is evident that the correlation functions (18) come from the Lagrangian (26). The Lagrangian is invariant under infinite number of infinitesimal transformations

$$
\delta_{c} \Phi^{a}=f_{c b}^{a} \Phi^{b}, \quad \delta_{c} \bar{\Phi}_{a}=f_{a c}{ }^{b} \bar{\Phi}_{b},
$$

From this invariance immediately follow the Wards identities for correlation functions (18). 


\section{Discussion and conclusion}

It is interesting to note that the interaction part of the Lagrangian (26) is nothing but the first three-linear term of generator $V\left(\eta, \frac{\partial}{\partial \eta}\right)$ of the homotopy Lie algebra. The homotopy Lie algebra [1] is described by the operator

$$
V=f_{c b}^{a} \eta^{c} \eta^{b} \frac{\partial}{\eta^{a}}+f_{c b d}^{a} \eta^{c} \eta^{b} \eta^{d} \frac{\partial}{\eta^{a}}+\ldots
$$

which satisfies the condition

$$
\{V, V\}=0
$$

We assume the identification

$$
\eta^{a} \sim \Phi_{a}, \frac{\partial}{\partial \eta^{a}} \sim \bar{\Phi}^{a}
$$

so the contractions are identical. This term is also the BRST charge for the group transformations (28).

We expect that leading singularities of N-point discrete states loop amplitudes coming from open string field theory [8] also reduce to the Lagrangian (26) with full coupling constant

$$
f_{a b}{ }^{c}=\sum_{\text {loop }}<Y_{a}^{+} Y_{b}^{+} Y_{c}^{-}>_{\text {loop }} .
$$

It is well known that one cannot limited by self-interacting open string and one has to introduce an open-closed transition. One can expect that for closed string due to [17] one has to introduces the Lagrangian

$$
\mathcal{L}_{\text {closed }}(\Theta, \bar{\Theta})=\sum_{a} \bar{\Theta}_{a} \Theta^{a}+g \sum_{a, b, c} \Theta^{a} \Theta^{b} \bar{\Theta}_{c} f_{a b}^{c}+g^{2} \sum_{a, b, c, d} \Theta^{a} \Theta^{b} \Theta^{c} \bar{\Theta}_{d} f_{a b c}{ }^{d}+\ldots,
$$

where

$$
f_{a b \ldots c}{ }^{d}=<O_{a} O_{b} \ldots O_{c} \bar{O}^{d}>.
$$

The interaction is nothing but the full Witten Zwiebach operator $V$. One can also say that the interaction part of the action (30) is the BRST charge for a non-closed algebra. The action is invariant under transformations

$$
\begin{aligned}
& \delta_{c} \Theta^{a}=f_{c b}{ }^{a} \Theta^{b}+f_{c b d}{ }^{a} \Theta^{a} \Theta^{d}+\ldots, \\
& \delta_{c} \bar{\Theta}_{a}=f_{a c}{ }^{b} \bar{\Theta}_{b}+f_{a c d}{ }^{b} \Theta^{d} \bar{\Theta}_{b}+\ldots,
\end{aligned}
$$

if we assume that formfactors are the subject of relations which follow from the nilpotency condition $\{V, V\}=0$.

It seems that discrete states in $2 \mathrm{D}$ gravity remind resonances in quantum theory. If one takes into account a perturbation of the model then for scattering amplitudes one would obtain an analogue of the Breit-Wiegner formula [18]. Note also that for analogues problem of small denominators in classical mechanics one should consider non-quadratic terms which drastically change the behaviour of trajectories [19]. Probably similar nonperturbative effects will play an important role in 2D string theory and we expect that a more suitable framework to describe them is string field theory. One can expect that the model exhibits a phase transition. One of phases is a topological one with scattering amplitudes described by the effective action (26). 
In summary, we have argued that under some renormalization procedure the scattering amplitudes of 2D open string discrete states reduce to those of the model with the Lagrangian (26).

\section{ACKNOWLEDGMENT}

The authors are grateful to P.B.Medvedev and I.V.Volovich for useful discussions.

\section{References}

[1] E.Witten and B.Zwiebach, preprint IASSNS-HEP-92/4 (1992)

[2] E.Verlinde, preprint IASSNS-HEP-92/5 (1992)

[3] D.Gross, I.Klebanov and M.J.Newman, Nucl. Phys. B350 (1991) 621 D.Gross and I.Klebanov, Nucl. Phys. B352 (1991) 671.

[4] A.Polyakov, Mod.Phys.Lett. A6 (1991) 635.

[5] B.Lian and G.Zukerman, Phys. Lett. 254B (1991) 417; Phys. Lett. 266B (1991) 21.

[6] D.Gross and I.Klebanov, Nucl. Phys. B359 (1991) 3.

[7] P.Bouwknegt, J.McCarthy and K.Pilch, CERN preprints, CERN-TH.6162/91 (1991); CERN-TH.6279/91 (1991).

[8] I.Ya.Aref'eva and A.V.Zubarev, Mod. Phys.Lett. (1992)

[9] S.Mukherji, S.Mukhi and A.Sen, Phys. Lett.266(1991)337.

[10] E.Witten, Ground ring of two dimensional string theory, preprint IASSNS-HEP-91/51.

[11] K.Itoh and N.Ohta, Preprint FERMILAB-PUB-91/228-T.

[12] L.Klebanov and A.M.Polyakov, Mod.Phys.Lett.A6(1991)3273.

[13] M.Li, Texas preprint, UCSBTH-91-47.

[14] J.Avan and A.Jevicki, Phys. Lett.66B(1991)35; D.Minic, J.Polchinskiand Z.Yang, Texas preprint, UTTG-16-91(1991); G.Moore and Seiberg, Rutgers preprint RU-9129(1991); S.Das, A.Dhar, G.Mandal and S.R.Wadia, Preprint IASSNS-HEP/91/52,91/72 and $91 / 79,(1991)$.

[15] L.Klebanov, Mod.Phys.Lett.A7(1992)723.

[16] J.Stasheff, preprint UNC-MATH-90/1.

[17] M.Saadi and D.Zwiebach, Ann.Phys.192(1989)213; M.Kaku, Phys.Rev.D41(1990)3734; T.Kugo and K.Suehiro, Nucl. Phys.B337(1990)434.

[18] L.D.Landau and E.M.Livshich , Quantum mechanics, Nauka, 1973.

[19] V.I.Arnold, Mathematical questions of classical mechanics, Nauka,1990. 